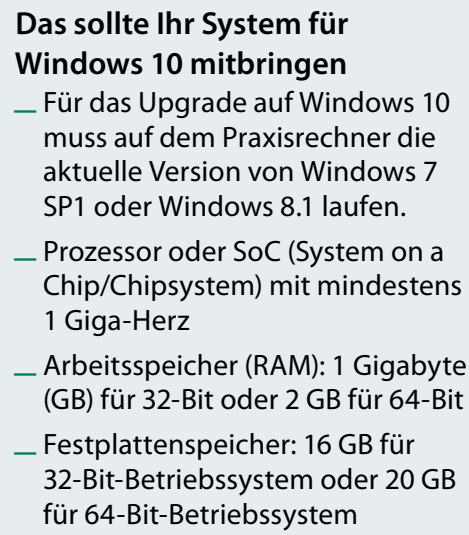

verstärkt auf seine Cloud-Lösung „One Drive“. Einzelne Anwendungen würden öfter das Verschieben von Daten in die Cloud anfragen - oder kommen eventuell schon mit entsprechender Voreinstellung daher.

Dadurch könnten möglicherweise Daten leichter ungewollt in Microsofts Cloud landen. CGM rät den Praxen daher, sich das neue Betriebssystem nur von erfahrenen und offiziellen Partnern der Systemhäuser einrichten zu lassen. Wobei: Um Zugriff auf die Microsoft Cloud zu haben, muss sich der Anwender zumindest einmalig auch dafür bei Microsoft registriert haben.

Was Praxen vor dem Umstieg aber unbedingt tun sollten: Prüfen, ob nicht nur ihr derzeitiges Betriebssystem, sondern auch die Hardware dafür überhaupt geeignet ist. „Unter anderem muss das Vorsystem Windows 7 SP1 oder Windows 8.1 Update sein“, so CGM. Und der Festplattenspeicher muss mindestens 16 Gigabyte (GB) für 32-BitBetriebssysteme oder 20 GB für 64-BitBetriebssysteme hergeben. Ärzte können sich auf der Internetseite von Microsoft über die weiteren Systemvoraussetzungen informieren.

\section{EDV-Häuser liegen noch im Zeitplan}

Ab wann die Praxissoftware tatsächlich problemlos unter Windows 10 läuft, erfahren Ärzte direkt von den SoftwareAnbietern. Dazu gebe es eine spezielle Benachrichtigung, heißt es bei CGM und medatixx. „Wir gehen davon aus, im Laufe des Jahres die Unterstützung von Windows 10 gewährleisten zu können“, berichtet medatixx. Damit können die Praxen das Upgrade zu Windows 10 dann immer noch kostenfrei in Anspruch nehmen - sofern sie bereits Windows 7 oder Windows 8 nutzen.

Seien alle Voraussetzungen für den reibungslosen Betrieb gegeben, macht der Umstieg nach Angaben der CGM auch tatsächlich Sinn: Denn Windows 10 sei in der Bedienung und Geschwindigkeit besser als die Vorgängerversion und die Praxen könnten so an aktuellen Sicherheitsfeatures teilhaben. Wobei das mit seiner Kacheloptik bislang auffälligste Windows-System - Windows 8 geschätzt gerade einmal von 5-8\% der Praxen, die mit einem System der CGM arbeiten, genutzt wurde. Rund 3-5\% der Praxen würden sogar noch, etwa wegen einer speziellen Geräteanbindung, mit Windows XP arbeiten, für das es seit dem Frühjahr 2014 keine SicherheitsUpdates mehr von Microsoft gibt. Auch von medatixx ist $\mathrm{zu}$ hören, dass der Großteil der Praxen, die ein System des IT-Anbieters nutzen, mit Windows 7 und nicht der neueren Version Windows 8 arbeitet.

RebekkaHöhl

\title{
Was kann Microsofts neues Betriebssystem?
}

\section{Windows 10 bietet Anwendern viele neue Funktionen. Dabei setzt Microsoft vor allem auf Sicherheit.}

$M$ it Windows 10 läutet Microsoft eine neue Ära ein. Erstmals stellt das US-amerikanische Unternehmen eine einheitliche Software-Plattform für alle Geräte bereit. Das heißt, das Betriebssystem läuft einheitlich auf PC, Notebook, Tablet und Smartphone. Es soll sogar für das Internet der Dinge, also etwa Anwendungen des Smart Living, geeignet sein. Das System passt sich automatisch an das jeweilige Gerät an und lässt sich je nach Bedarf mit Tastatur, PC-Maus oder per Fingerstreich bedienen.

Wichtig ist für Nutzer vor allem, dass die Schwachstellen des Windows 8 ausgeräumt wurden. Dort musste etwa auf das Anzeigen von RSS-Feeds im Web-
Browser teils verzichtet werden, Windows-Einstellungen wurden an verschiedenen Orten des Systems abgelegt, und allem voran erschwerte die Kacheloptik das Arbeiten mit dem System.

Da ist es erfreulich, dass mit Windows 10 das altbekannte Startmenü wieder zurückkehrt. Wie in den früheren Versionen gelangt der Nutzer auch hier mit einem Klick zu den gewünschten Funktionen und Anwendungen.

Außerdem zieht mit „Cortana“ Microsofts neue persönliche digitale Assistentin auf PC und Tablet ein. Über sie lässt sich der Rechner mittels Sprache steuern. Und auch am Browser hat Microsoft nachgebessert: Der Internet Explorer weicht Microsoft Edge, bei dem Anwender die Möglichkeit haben, via Tastatur oder Stift direkt Kommentare auf einer Website einzugeben und diese mit anderen zu teilen.
Interessant für Praxen wird sein, dass die Unternehmensvariante von Windows 10 neben zusätzlichen Sicherheitsfeatures auch ein Gerätemanagement bietet, das immer dann sinnvoll ist, wenn neben den festen Rechnern in der Praxis mobile Geräte wie Tablets im Rahmen der ärztlichen Tätigkeit im Einsatz sind. So lassen sich spezielle zeitliche Wartungsfenster für das Aufspielen von Updates auf einzelnen Geräten einrichten.

Für die Datensicherung, falls ein mobiles Gerät abhanden kommt, hat sich Microsoft gleich mehrere Sicherungswege überlegt: Windows 10 bietet etwa die Möglichkeit einer Zwei-Faktor-Authentifizierung. Hierbei melde sich der Nutzer mit einem Code, einem biometrischen Identifizierungsmerkmal oder einem zweiten Gerät bei einem über Active Directory oder Azure Active Directory geschützten Container an, der $\mathrm{Zu}$ gangsdaten zu unterschiedlichen Systemen biete, heißt es. Rebekka Höhl/dpa 